\title{
Dynamics of Vertical Axis Wind Turbines (Darrieus Type)
}

\author{
A.F. ABDEL AZIM EL-SAYED \\ Mechanical Engineering Department, Zagazig University, Zagazig, Egypt \\ C. HIRSCH and R. DERDELINCKX \\ Vrije Universiteit Brussel, Dept. of Fluid Mechanics, Pleinlaan 2,1050 Brussel, Belgium
}

\begin{abstract}
A computing package that combines finite element methods for evaluating the resonance frequencies and modes of turbine subcomponents (blade, tower and shaft) together with the aerodynamic calculations for forces and moments taking into consideration the dynamic stall as well as the dynamic response is developed. This method was applied to a realistic VAWT namely; the PIONEER I built in the Netherlands by Fokker company. A reasonable agreement between the calculated and field results was predicted.
\end{abstract}

Key Words: Vertical axis; Darrieus; Aerodynamics; Aeroelasticity; Dynamics

\section{INTRODUCTION}

$\mathbf{T}$ To meet economic levels of power generation, the wind turbine designer is being forced towards compliant designs. As a consequence of this fact, more emphasis is being placed on both aeroelastic and dynamic analyses in the design phase of producing a new product. Since a wind turbine blade is highly dynamically loaded, it requires intensive investigations before being put into production to ensure both safety and reliability. This paper is devoted towards the investigation of the aerodynamics and stability of a Darrieus vertical axis wind turbine (VAWT). VAWT's have several advantages over the horizontal axis wind turbines; (HAWT's) like the indifference to wind direction, the proximity of the gearbox and generator to the ground and the non-reversal gravitational stresses in their troposkien shaped blades. However, the main disadvantage of VAWT's is their disabilities for self starting.

At the Vrije Universiteit Brussel (V.U.B) a substantial effort has been done to investigate both the aerodynamics and dynamics/aeroelasticity of both HAWT's and VAWT's. Concerning the specific work with VAWT's, a computing package is developed which combines the versatile finite element methods (FEM) for evaluating the natural frequencies and modes of the turbine's subcom- ponents; Bathe [1982], modal coupling technique; Benfield, et al. [1971], to define the behavior of the whole structure, the aerodynamic calculations for loads on blades, eigenvalue problem solver for natural frequencies and modes evaluation as well as a stability investigation via a forced vibration analysis. This package is analogous to that developed by Garrad, et al. [1984]. In this regard it can substitute using the well-known code NASTRAN (MSA/NASTRAN User's manuals [1981]), and also the European code ARLIS, Kirchgäßner [1984]. Extensive analytical and experimental research works regarding the aerodynamics, aeroelasticity and dynamics of Darrieus VAWT's are performed both in Sandia laboratories; Lobitz [1986] and Popelka [1982], in USA and in the Netherlands Energy Research Foundation (ECN) in Netherlands; Machielse [1984] and Machielse, et al. [1986]. Most investigations elsewhere compare their results with the findings published by Sandia and/or ECN. In this paper, our model is applied to the $15 \mathrm{~m}$ VAWT manufactured by ECN and named Pioneer I, Ottens [1981].

\section{MATHEMATICAL MODEL}

A mathematical model capable for investigating both of the aeroelastic and dynamic responses of a Darrieus 
VAWT is presented here. The equations of motion for a small displacement (q) from a steady state for the discretized model may be derived either from the energy equation of continuum mechanics, or the principle of virtual displacements leading to a Lagrangian form of governing equation. Both techniques lead to the same equation. The following assumptions are made:

1. The blade has a troposkien shape; refer to Blackwell, et al. [1974].

2. Blade cross section is symmetric about its major principal axis and can have three distinct points; namely, the elastic, aerodynamic and tension centers. The elastic and mass centers are coincident.

3. Blade material is isotropic and linearly elastic.

4. Blade cross sections which are normal to the elastic axis before deformation remain plain after deformation (Bernoulli-Euler hypothesis). Also torsional warping is negligible.

5. Blade can bend in two mutually perpendicular directions normal to the elastic axis.

6. Deflections, strains and rotations are small.

7. Quasi-steady blade-element strip theory is applicable.

\section{Frames of Reference}

The first frame of reference is an inertial one So(Xo, Yo, Zo) fixed to the undeflected rotor. Next a series of transformations including rotations and translations are considered. $\mathrm{S} 1(\mathrm{X} 1, \mathrm{Y} 1, \mathrm{Z} 1)$ has the same origin but rotated an angle $\theta$ w.r.t. the inertial frame So. Next $\mathrm{S} 2(\mathrm{X} 2, \mathrm{Y} 2, \mathrm{Z} 2)$ is shifted a distance $\delta$ from the frame $\mathrm{S} 1$. While S3(X3, Y3, Z3) and S4(X4, Y4, Z4) are inclined angles $\phi$ and $\psi$ w.r.t. the $\mathrm{Y} 2$ and $\mathrm{X} 3$ respectively. Finally the frame S5(X5, Y5, Z5) locates any point on the blade itself whose tangent is inclined angle $\gamma$ to the Z4 direction. The different frames of reference are related to each other by the equation.

$$
\begin{aligned}
& \{\mathrm{Si}\}=[\mathrm{Ki}]\{\mathrm{Si}-1\}, \quad \mathrm{i}=1, \ldots, 5 \\
& \text { and }\left\{\mathrm{S}_{\mathrm{i}}\right\}^{\mathrm{T}}=\left\{\mathrm{X}_{\mathrm{i}} \mathrm{Y}_{\mathrm{i}} \mathrm{Z}_{\mathrm{i}}\right\}
\end{aligned}
$$

and $\{\mathrm{Ki}\}$ is a transformation matrix. More details are given in El-Sayed [1990]. Figure (1) illustrates these frames of reference.

\section{Finite Element Method}

Treatment of such flexible continuous body can be performed by considering its deformation as the sum of few number of its normal modes multiplied by generalized coordinates. This method is known as the MODAL METHOD. This method is advantageous over the lumped mass deformation as in the former only few
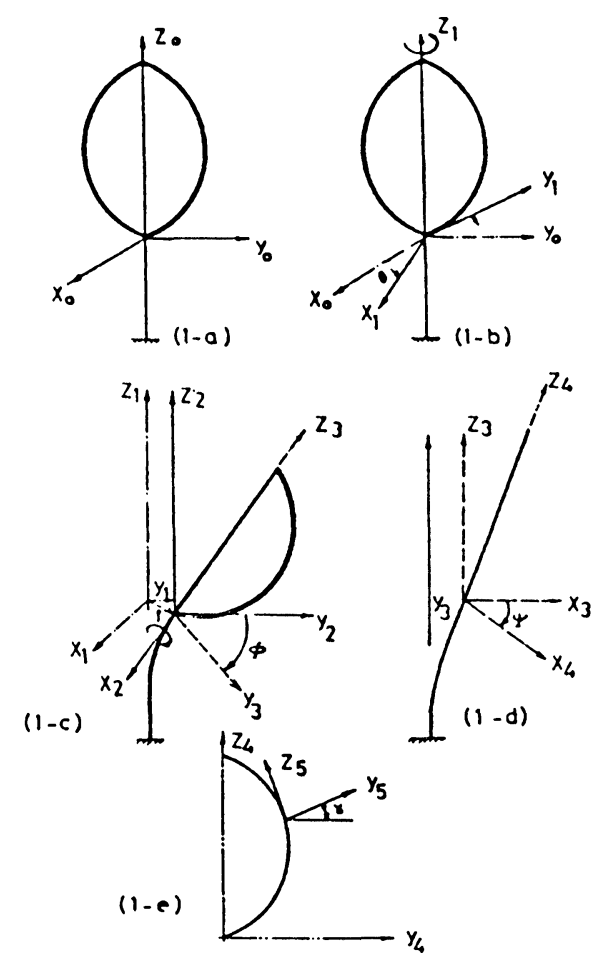

FIGURE 1 Different frames of reference.

number of the generalized coordinates or in other words unknowns are assigned. Usually, vibration modes of non-rotating turbines are adopted. To calculate these vibration modes, finite element methods are applied. Vibration modes of the components of the whole structure; here the blades, tower and the shaft together with the transmission and generator, are calculated. This is described as the "component mode method".

The subcomponents of the wind turbine are discretized into finite number of beam elements. Each beam element is characterized by two nodes and 12 degrees of freedom (6 rotational and 6 translational). The coordinates of each node together with the cross-sectional area and moments of inertia about three mutually perpendicular axes and the material density distribution are fed to a structural code. Consequently, the mass, flexural and torsional rigidities of each element are calculated. A powerful code developed by the structural department of the Vrije Universiteit Brussel; Belgium, known as DAPST (which is analogous to NASTRAN) is used to calculate the natural frequencies and modal shapes of the three subcomponents (refer to its user's guide [1985]).

Since blades are axisymmetric, then the same mode shape can be used for all blades. The displacement and/or rotation of a point on the kth blade and tower are denoted by Sik and qj; where $i$ varies from 1 to $n$ and $j$ varies from 1 to $\mathrm{m}$. The generalized coordinates of the shaft are its angular displacement $(\Delta \theta)$, and the torsional deflection 
of the mechanical transmission and rotating part of the generator $(\Delta \theta 2)$.

\section{Equations of Motion in Lagrangian Form}

Subsequent to these steps the kinetic and potential energies are derived as described in [12]. Since the governing equations in the Lagrangian form have the form:

$$
\frac{\mathrm{d}}{\mathrm{dt}}\left(\frac{\partial \mathrm{T}}{\partial \dot{\mathrm{h}}_{\mathrm{i}}}\right)-\frac{\partial \mathrm{T}}{\partial \mathrm{h}_{\mathrm{i}}}+\frac{\partial \mathrm{V}}{\partial \mathrm{h}_{\mathrm{i}}}+\frac{\partial \mathrm{F}}{\partial \dot{\mathrm{h}}_{\mathrm{j}}}=\mathrm{Q}_{\mathrm{i}}
$$

$\mathrm{i}=1,2, \ldots \mathrm{n}$

where $\mathrm{T}$ and $\mathrm{V}$ are respectively the kinetic and potential energies of the whole turbine, while hi are the generalized coordinates $(\Delta \theta, \Delta \theta 2, \mathrm{qj}, \mathrm{Sik})$. Forces are divided into conservative and non-conservative forces. Here both of the aerodynamic and gravitational forces represent the conservative forces. These conservative forces are derived from the potential energy. The non-conservative forces (except for the viscous damping) are expressed by Qi. The viscous damping are expressed by $\partial \mathrm{F} / \partial \mathrm{hi}$ where $\mathrm{F}$ is the well known Rayleigh's Dissipation Function (Strickland [1975]).

\section{Aerodynamic Force}

The aerodynamic forces are defined both theoretically (refer to Strickland [1975], Sharpe [1977], Templin [1974], Simhan [1984] and Mandal [1986]) and experimentally (refer to Worstell [1979], Muraca, et al. [1976] and South, et al. [1972]). The double-multiple stream tube theory $[23,24]$ is employed in this model. In such a model the Darrieus VAWT is represented by a pair of actuator disks in tandem at each level of the rotor as illustrated in Figure (2). The elements of normal and tangential forces $(\delta \mathrm{Nk}$ and $\delta \mathrm{Tk})$ and the resulting moment on a strip ( $\delta \mathrm{Mk})$ through the principle of virtual work provide the generalized forces $\mathrm{Q}_{\Delta \theta}$, Qqi and Qski. Dynamic stall will be discussed later on.

\section{Gravitational Force}

The forces due to gravity are the second conservative force. The generalized forces due to gravity associated with the tower and blades are, $\mathrm{G}_{\Delta \theta}$, Gqi and GSik respectively

\section{Modal Coupling}

To apply the modal coupling technique; Benfield, et al. [1971], the generalized coordinates are assembled into the vector $\bar{y}$ which includes $(\Delta \theta, \Delta \theta 2, \mathrm{qj}$, Ski $), \mathrm{k}=$ number of blades.

\section{Equations of Motion}

After a lengthy mathematical deductions, equation (2) will have the form:

$$
\begin{aligned}
& {[\mathrm{m}] \overline{\mathrm{y}}+([\mathrm{d}]+\Omega[\mathrm{g}]) \overline{\dot{y}}+\left([\mathrm{k}]-[\mathrm{Gr}]+\Omega^{2}[\mathrm{~s}]+\right.} \\
& \left.\Omega^{2}[\mathrm{c}]\right) \overline{\mathrm{y}}=\overline{\mathrm{Q}}
\end{aligned}
$$

where

$\overline{\mathrm{Q}}=\left[\begin{array}{lllll}\mathrm{Q}_{\theta} & 0 & \mathrm{Q}_{\mathrm{T}} & \mathrm{Q}_{\mathrm{B} 1} & \mathrm{Q}_{\mathrm{B} 2}\end{array}\right]^{\mathrm{T}}$

and $[\mathrm{m}],[\mathrm{d}],[\mathrm{k}]$ are the structural mass, damping and stiffness matrices respectively. [g], [s] are the coriolis (gyroscopic) and geometric stiffness matrices. [Gr], [c] are the gravitational and centrifugal matrices.

Moreover, $\bar{Q}$ represents all the forces, which is now a function of both space and time (due to the aerodynamic forces on blades). Calculations are now performed in the time domain using the classical 4th order Runge-Kutta integration scheme, with automatic time step integration.

\section{Aerodynamics with Dynamic Stall Calculations}

As was pointed out earlier, the aerodynamic forces are calculated with the double multiple streamtube

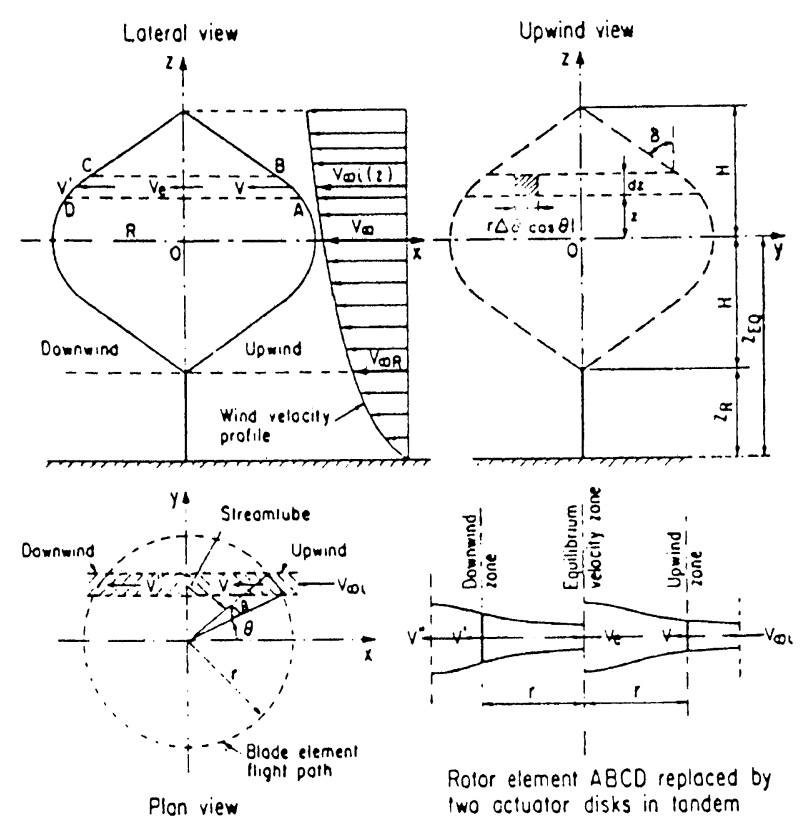

FIGURE 2 Darrieus rotor geometry and the double-multiple stream tube. 
theory. At higher wind speed this theory fails to predict the forces on the turbine correctly due to dynamic stall effects. Therefore, the present theory has been extended, to account for these effects. The circular path of the blades of a vertical axis wind turbine cause periodic oscillations of the angle of attack at a frequency of once per revolution and at higher multiples of this frequency. This rapid variation introduces unsteady flow phenomenon, that are not accounted for by the classical theories. They are known as dynamic stall. Stall is a phenomenon that occurs at higher angles of attack (of the order of 10 degrees) when the flow separates from the body. As a result a pressure drop and thus a decrease in lift will occur. When this occurs at a relatively slow angle of attack change, the phenomenon is called "static stall". When the angle of attack changes more rapidly unsteady flow phenomenon introduces hysteresis effects as shown in figure 3 . When the angle of attack increases rapidly above the static stall angle, the separation of the flow is retarded and stall will occur at higher angles of incidence. The lift forces will be higher than in the static case. In order to take into account dynamic stall effects, the Boeing-Vertol dynamic-stall model, modified by Strickland [1975], is used. This model assumes that the lift-curve slope and the zero-lift angle remain unchanged and that the only change due to dynamic effects is the angle of attack at which stall occurs. Thus a modified angle of attack is utilized for use in entering twodimensional force coefficient data.

In this method dynamic stall corrections are considered only for angles of attack above 5 degrees and below three times the static stall angle. Lift and drag coefficients are calculated first for the static case and then adjusted for dynamic stall effects. A modified angle of attack $\alpha \mathrm{m}$ is calculated as:

$$
\alpha_{\mathrm{m}}=\alpha_{0}-\mathrm{G} \cdot \Delta \alpha \cdot \mathrm{S}_{\alpha}
$$

where:

$$
\Delta \alpha=\mathrm{K} .\left[\alpha . \mathrm{C} /\left(2 \mathrm{U}_{\infty}\right)\right]^{0.5}+\mathrm{K}_{1}
$$

$\mathrm{K}, \mathrm{K} 1$ are empirical constants, depending on the Mach number and the thickness ratio of the profile. For the
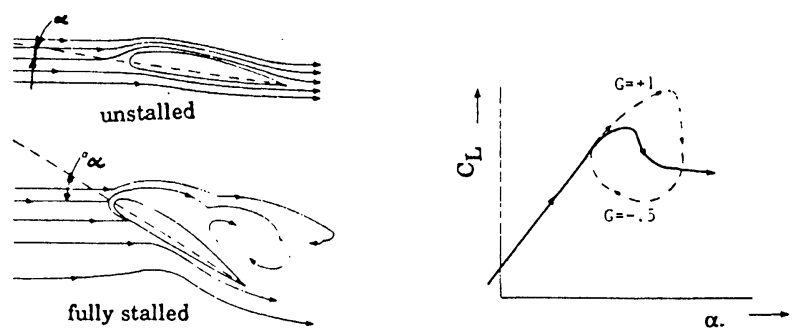

FIGURE 3 Lift force and weighing factor for hysteresis loop.
NACA 0012 profile the actual values are $\mathrm{K}=74.5$, $\mathrm{Kl}=4.47 . \mathrm{G}$ is a weighing factor depending on which part of the dynamic stall hysteresis loop, the cycling value of $\alpha \mathrm{m}$ lies. (See Figure 3).

The rate of change $\alpha$ is determined as:

$$
\begin{aligned}
& \frac{\mathrm{d} \alpha}{\mathrm{dt}}=\frac{\Omega \cdot \cos \phi}{\left[1+\left(\frac{\sin \theta \cdot \cos \phi}{\frac{\mathrm{r}}{\mathrm{R}} \cdot \frac{\mathrm{R} \cdot \Omega}{\mathrm{V}_{\mathrm{a}}}+\cos \theta}\right)^{2}\right] \cdot\left[\frac{\mathrm{r}}{\mathrm{R}} \cdot \frac{\mathrm{R} \cdot \Omega}{\mathrm{V}_{\mathrm{a}}}+\cos \theta\right]} \\
& \cdot\left\{\frac{\sin \theta}{\frac{\mathrm{r}}{\mathrm{R}} \cdot \frac{\mathrm{R} \cdot \Omega}{\mathrm{V}_{\mathrm{a}}}+\cos \theta}+\cos \theta\right\}^{2}
\end{aligned}
$$

Once the modified angle of attack is calculated, the lift force coefficient CLs is calculated for stalled conditions. Finally the modified lift force coefficient is calculated from the static value CLo and the stall value CLs as:

$$
\mathrm{C}_{\mathrm{L}}=\mathrm{C}_{\mathrm{L} 0} \cdot(1-\mathrm{P})+\mathrm{C}_{\mathrm{Ls}} \cdot \mathrm{P}
$$

where: $\mathrm{P}$ is defined in figure 4.

\section{RESULTS}

In this section the dynamic response of blades and tower of the Pioneer I to the external forces is presented. These external forces are the centrifugal, gravitational and aerodynamic forces.

\section{CASE STUDY}

The mathematical model described above is now applied to one of the most documented wind turbines, namely PIONEER I, built in the Netherlands by Fokker company. Figure 5 illustrates this turbine and the employed

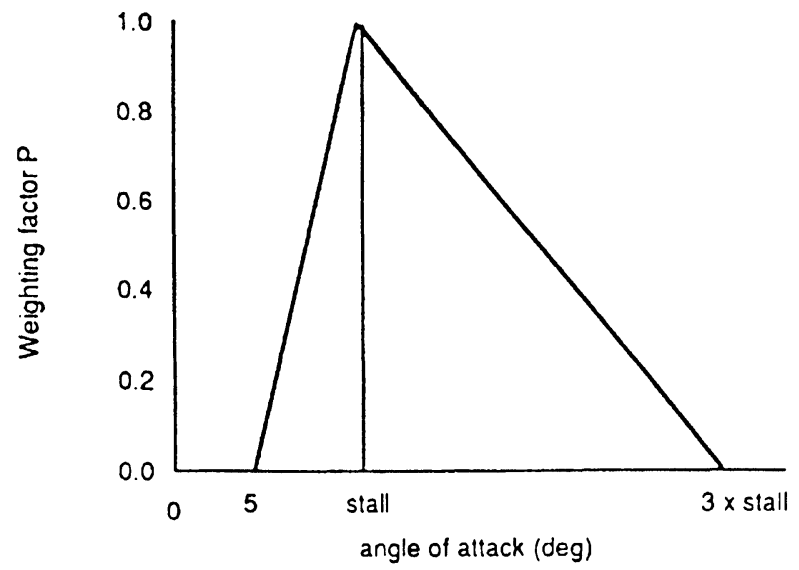

FIGURE 4 Weighing factor $P$. 


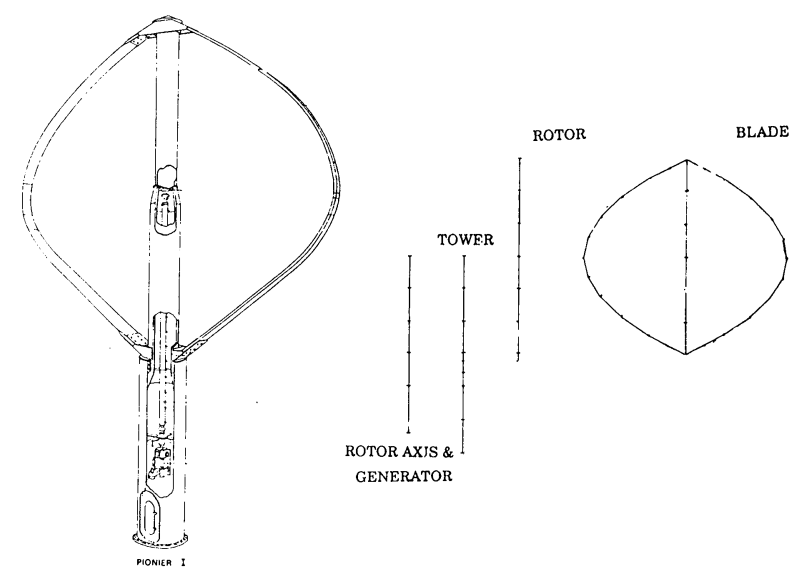

FIGURE 5 Pioneer I VAWT \& Discretized model (blade-rotortower-rotor axis \& generator).

discretized model. PIONEER I is a two-bladed VAWT with rotor height of $15 \mathrm{~m}$ and diameter of $14.92 \mathrm{~m}$, has a troposkien blade shape with a blade chord of $0.75 \mathrm{~m}$. The main bearings on the top of the tower and in the rotor center are situated at the inside of the rotor tube. Both of the rotor tube and the turbine tower are made of two steel tubes of different length, diameter and wall thickness. The blades have a NACA 0012 cross section and made of glass fiber reinforced polyester (GFRP) skins and a polyurethane foam core. Operational speed varies from 25 to $50 \mathrm{rpm}$ at a wind speed of $10 \mathrm{~m} / \mathrm{s}$. Power output at $18 \mathrm{~m} / \mathrm{s}$ is $65 \mathrm{~kW}$.

\section{Forces and Power}

Figure 6 shows the power coefficient versus wind speed for 30 and $50 \mathrm{rpm}$. (without dynamic stall). At $30 \mathrm{rpm}$ a maximal power is extracted at a wind speed of $7 \mathrm{~m} / \mathrm{s}$. This power is approximately $10 \mathrm{~kW}$. At $50 \mathrm{rpm}$ the power increases to $53 \mathrm{~kW}$ at a wind speed of $12 \mathrm{~m} / \mathrm{s}$. In figure 7 the power at a rotational speed of $40 \mathrm{rpm}$ is shown, as calculated without and with dynamic stall. It may be seen

PIONEER I Cp for 30 and $50 \mathrm{rpm}$

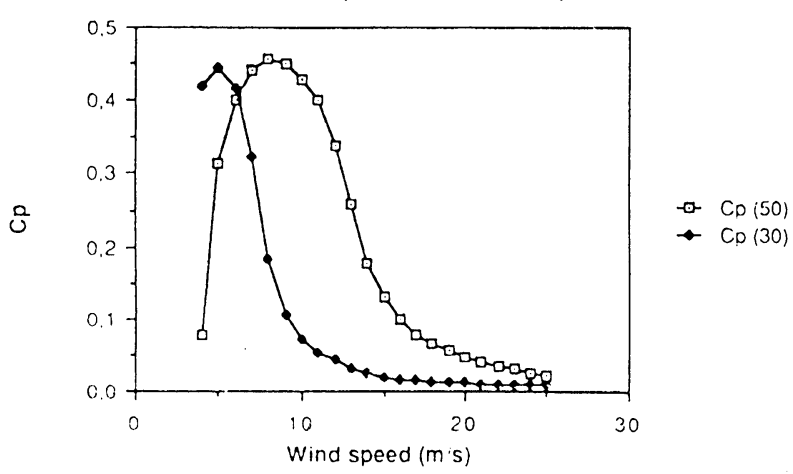

FIGURE 6 Power coefficient vs. wind speed (without dynamic stall).

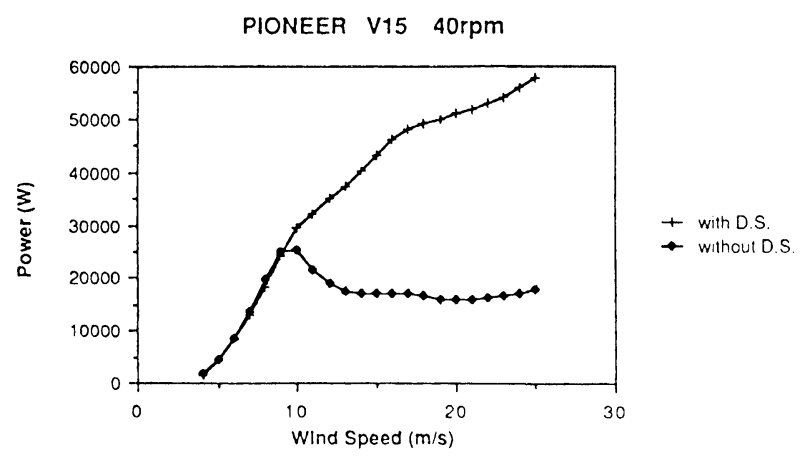

FIGURE 7 Power versus wind speed for a rotational speed of 40 rpm.

that for higher wind speeds the aerodynamic power is considerably higher when dynamic stall is taken into account.

The normal and tangential forces on the blades as a function of azimuthal position at a rotational speed of 50 $\mathrm{m} / \mathrm{s}$ and a wind speed of $10 \mathrm{~m} / \mathrm{s}$ are presented in figures 8 and 9 respectively. At the upstream side these forces are considerably larger than at the downstream side.

\section{Dynamic Response}

In figures 10 to 12 the generalized forces and dynamic blade responses due to the centrifugal forces are shown for a two mode representation of the blades. As can be seen from figure 10 the centrifugal force is constant and thus leads to a constant generalized force. In principle, the centrifugal force is balanced by the gravitational forces, due to the special geometry of a troposkien shape. This leads to a general force, that should be equal to zero. However due to numerical rounding errors, a small force remains that will result in a very low dynamic response, as can be seen in figures 11 and 12. This response is characterized by a damped oscillation level at transient, and a constant level after some settling time. This type of response is typical for a damped oscillation to a constant force.

In figures 13 to 15 the dynamic response of the blade to the gravitational forces are shown. As the centrifugal forces and gravity introduce a constant force into the blades of the turbine and into the tower, it will introduce a damped oscillation with constant level in the flapping direction. In lagging direction a zero displacement level should be found. However, due to the modal approximation and rounding errors, a small amplitude of the order of 10-6 remains.

In figures 16 to 18 the displacements of the midpoint of the blade and the generalized forces, due to aerodynamic loading are presented. As can be seen the aerody- 


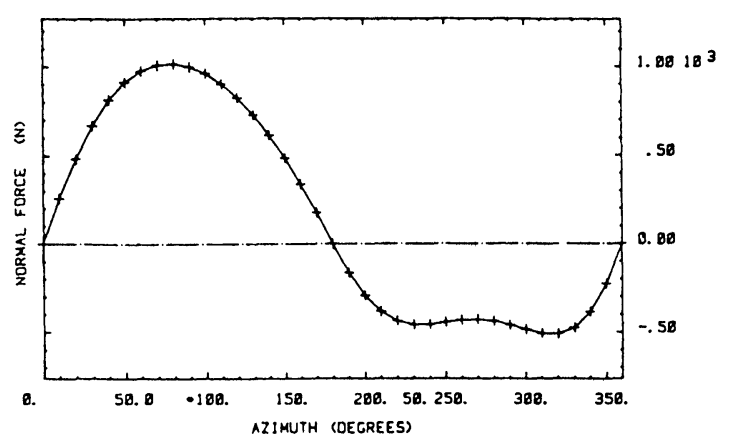

FIGURE 8 Normal force on the blade at wind speed of $10 \mathrm{~m} / \mathrm{s}$ and rotational speed of $50 \mathrm{~m} / \mathrm{s}$.

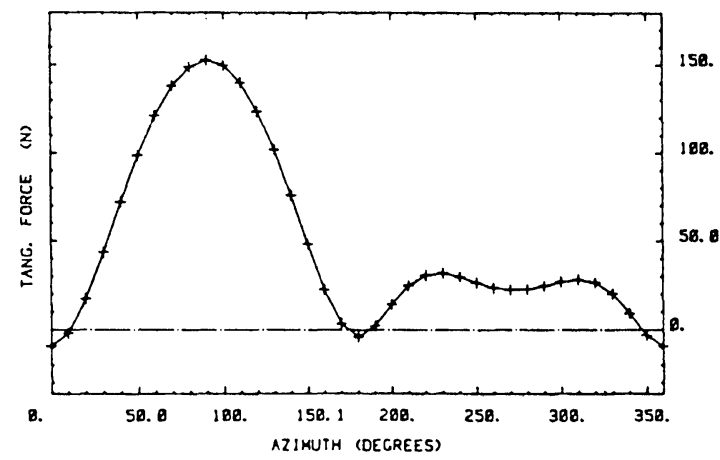

FIGURE 9 Tangential force on the blade at wind speed of $10 \mathrm{~m} / \mathrm{s}$ and rotational speed of $50 \mathrm{~m} / \mathrm{s}$.

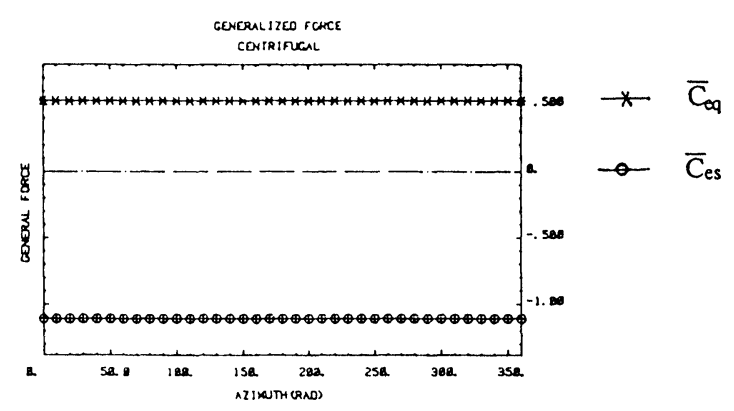

FIGURE 10 Generalized forces on the blade due to centrifugal forces.

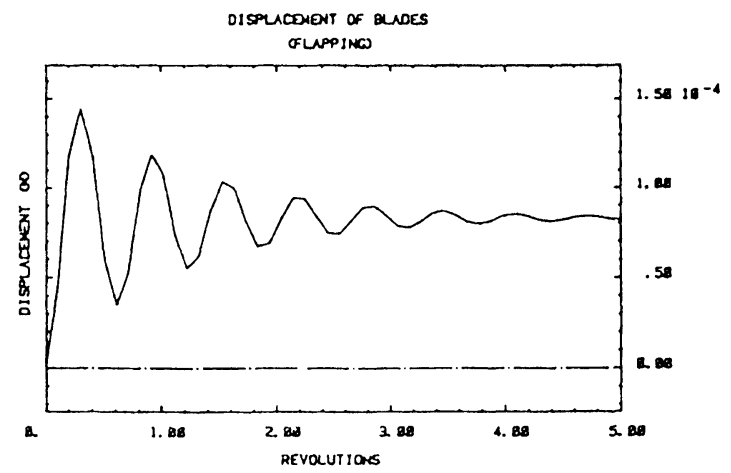

FIGURE 11 Flapping displacement of the centre of the blade due to centrifugal forces.

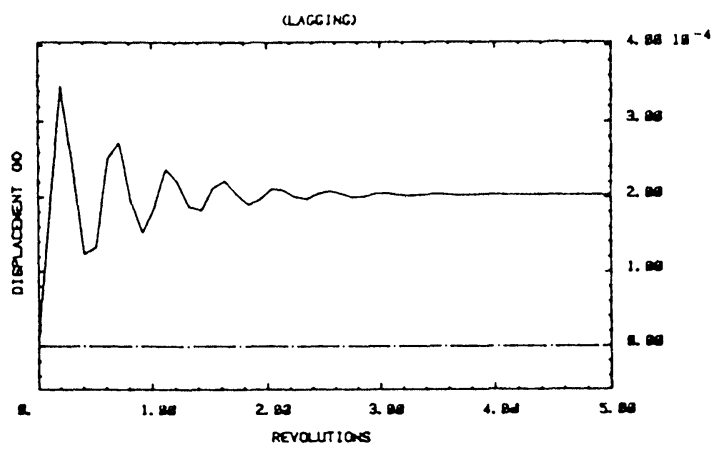

FIGURE 12 Lagging displacement of the centre of blade due to centrifugal forces.

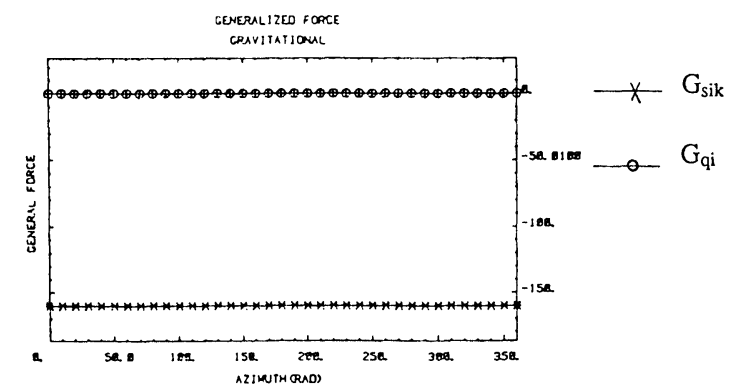

FIGURE 13 Generalized forces due to gravitational forces.

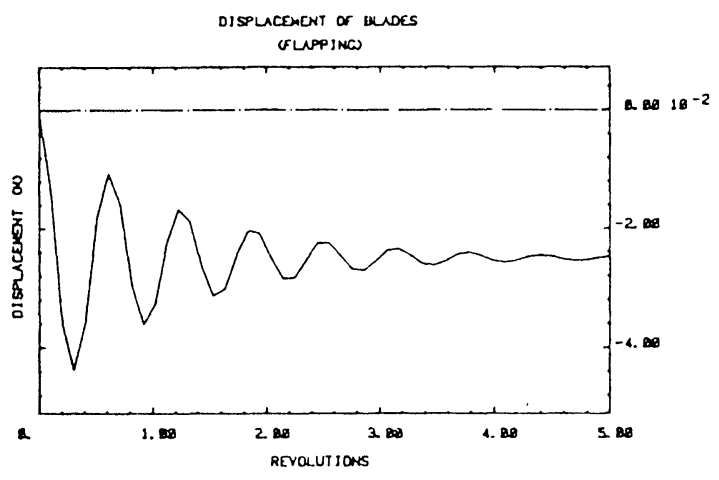

FIGURE 14 Flapping displacement of the centre of the blades due to gravitational forces.

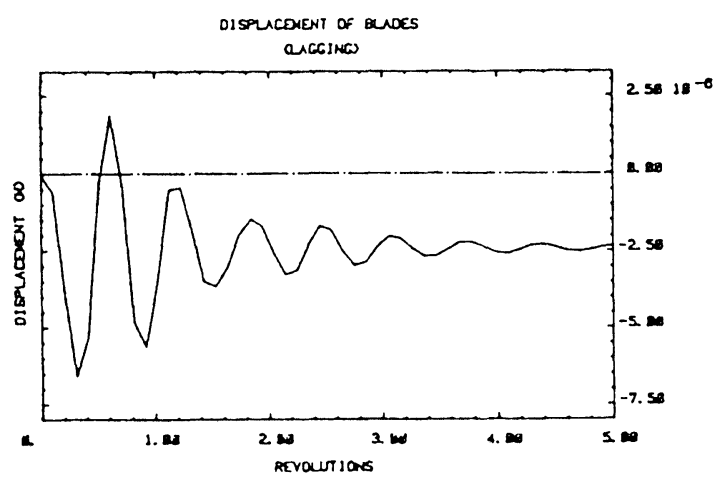

FIGURE 15 Lagging displacement of the centre of the blade due to gravitational forces. 


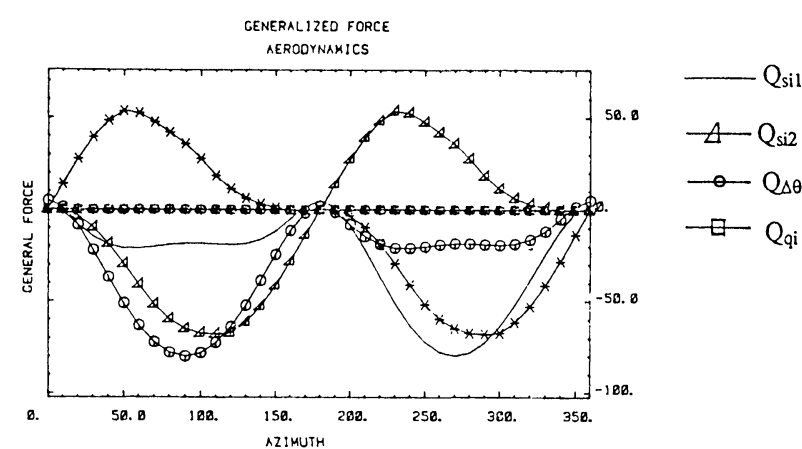

FIGURE 16 Generalized forces on the blades due to aerodynamic loading.

namic loading is more or less periodic, with a maximal amplitude when the blade is in the upstream region. For a damping ratio of 0.1 , the flapping displacement of the blade is symmetric, with a nearly zero amplitude at mid span. The lagging displacement follows the pattern of the aerodynamic forces with a period of 2 per revolution.

In figures 19 to 21 the generalized forces and displacements of the blade to all external loading are presented. As can be seen, the displacements of the midpoint of the blade in flapping motion are dominated by the gravitational forces, while the displacements in lagging motion are mainly due to the aerodynamic loading.

\section{CONCLUSIONS}

1. The designed package which is described here predicted its validity and accuracy in evaluating the aerodynamic, aeroelastic and dynamic behaviors of Darrieus VAWT's.

2. Dynamic stall has to be incorporated in the aerodynamic calculations since it drastically changes the extracted power compared to models ignoring it.

3. Centrifugal forces on blades are counteracted by gravitational ones. The transient behavior due to them shows a damped response.

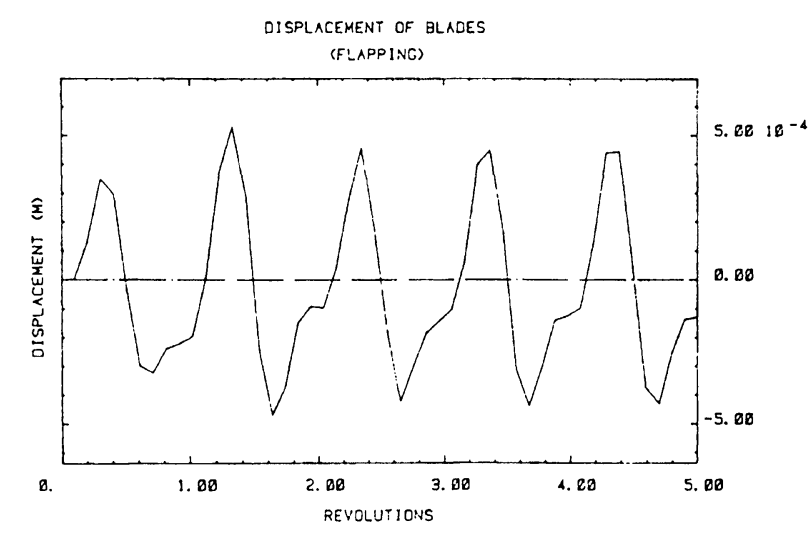

FIGURE 17 Flapping displacement of the centre of the blade due to aerodynamic loading.

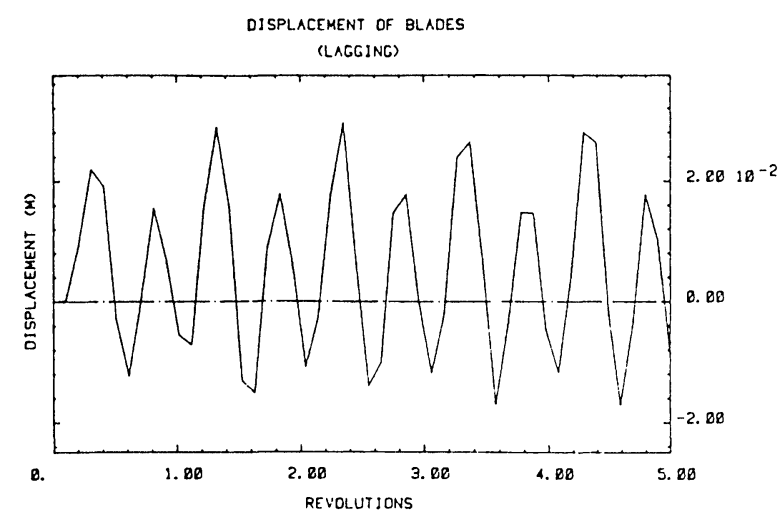

FIGURE 18 Lagging displacement of the centre of the blades due to aerodynamic loading.

4. Aerodynamic loading is periodic with maximum amplitude when blades are in the upstream region.

5. Displacements in the lagging and flapping directions are dominated by aerodynamic and gravitational forces respectively.

\section{ACKNOWLEDGEMENT}

The first author acknowledges the Financial support given to him by Vrije Universiteit Brussel while he was visiting professor to the fluid mechanics department in 1986.

The support of Mr. Riad M. El-Sharawi; Enppi Co., during the preparation of this paper is greatly acknowledged.

\section{Nomenclature \\ [c] : $\quad$ centrifugal matrix \\ C : $\quad$ cord length (m) \\ $C_{L} \quad: \quad$ modified lift forces coefficient \\ $C_{L o} \quad: \quad$ lift force coefficient for static case \\ $C_{L s} \quad: \quad$ lift force coefficient for stalled case \\ [d] : $\quad$ structural damping matrix}

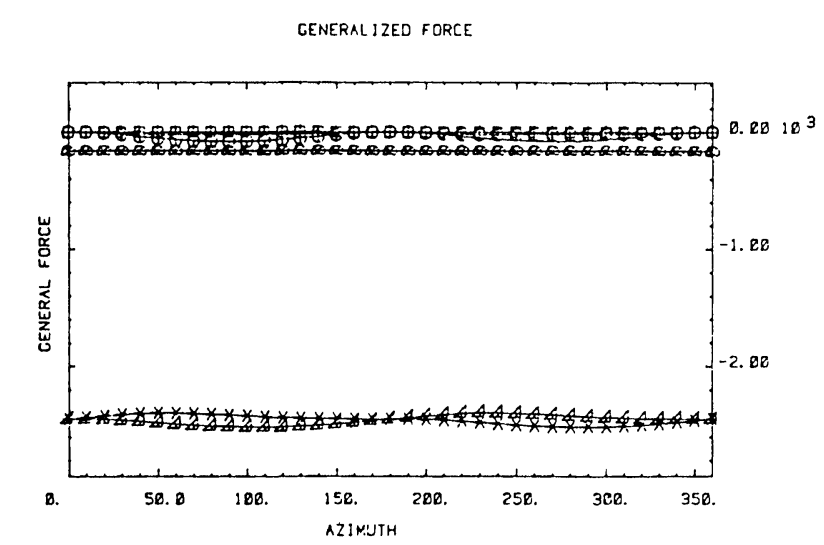

FIGURE 19 Generalized forces on the blades due to all loading. 


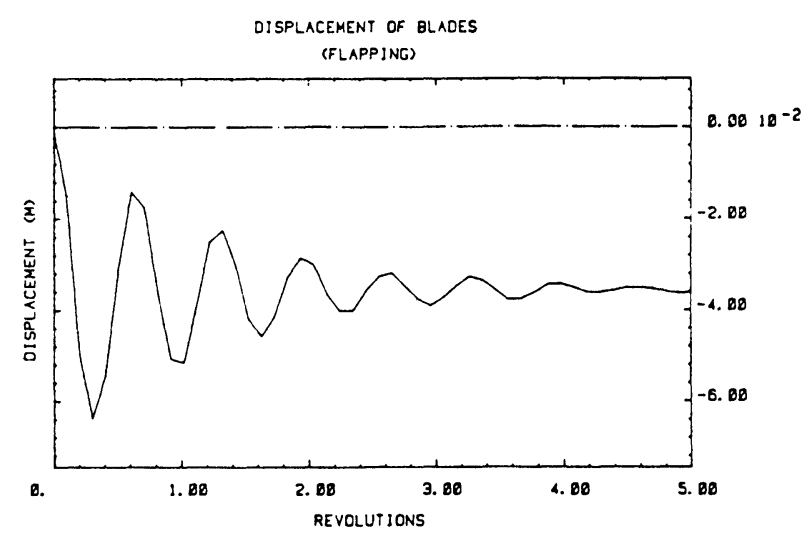

FIGURE 20 Flapping displacement of the centre of the blade due to all loading.

$\begin{array}{lll}F & : & \text { Rayleigh's dissipation force } \\ {[g]} & : & \text { gyroscopic stiffness matrix } \\ G & : & \text { weighing factor } \\ {[G r]} & : & \text { gravitational matrix } \\ h_{i} & : & \text { generalized coordinates } \\ {[k]} & : & \text { structural stiffness matrix } \\ K, K l & : & \text { empirical constants } \\ {\left[k_{i}\right]} & : & \text { transformation matrix } \\ {[m]} & : & \text { structural mass matrix } \\ P & : & \text { weighing factor (figure 4) } \\ Q_{i} & : & \text { non-conservative forces (except viscous } \\ & & \text { damping) } \\ Q & : & \text { force vector } \\ r & : & \text { local radius of blade (m) } \\ R & : & \text { blade radius at mid span (m) } \\ {[s]} & : & \text { geometric stiffness matrix } \\ S_{i} & : & \text { frame of reference } \\ S \alpha & : & \text { sign of the rate of change of } \alpha \text { (eqn 4) } \\ T & : & \text { kinetic energy } \\ U \propto & : & \text { wind speed (m/s) } \\ V & : & \text { potential energy } \\ V a & : & \text { local "disk" velocity (m/s) } \\ X, Y, Z & : & \text { cartesian coordinates }\end{array}$

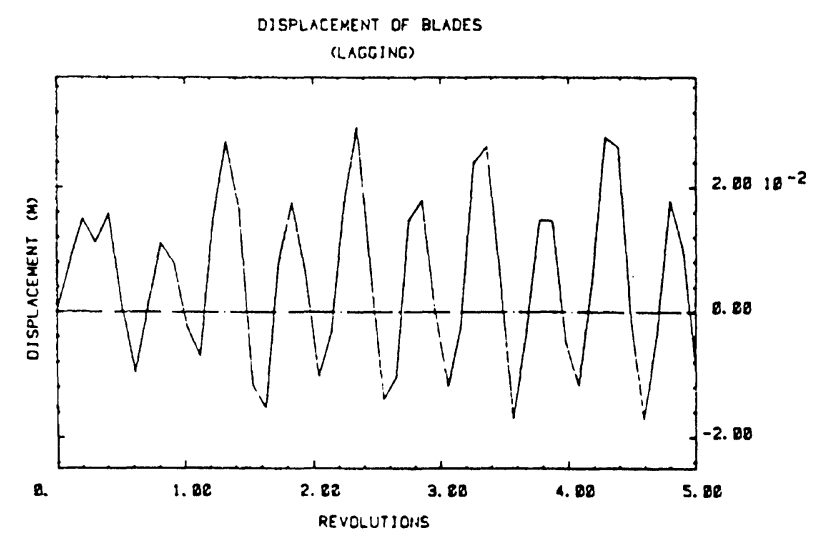

FIGURE 21 Lagging displacement of the centre of the blade due to all loading.

\begin{tabular}{|c|c|c|}
\hline $\bar{y}$ & $:$ & generalized coordinates vector \\
\hline$\delta M$ & : & $\begin{array}{l}\text { resulting moment on a blade strip element of } \\
\text { normal force }\end{array}$ \\
\hline & & element of normal force \\
\hline & & element of tangential force \\
\hline
\end{tabular}

\section{Greek Letters}

$\begin{array}{ll}\alpha & : \\ \alpha_{o} & : \\ \alpha_{m} & : \\ \gamma & : \\ \theta & : \\ \phi & : \\ \psi & : \\ \Omega & : \\ \Theta & : \\ \Phi & :\end{array}$

\author{
angle of attack \\ static angle of attack \\ modified angle of attack \\ angle between tangent to blade and $\mathrm{Z} 4$ \\ direction \\ rotational angle of S1 Frame w.r.t. $\mathrm{S}$ \\ rotational angle of S3 Frame w.r.t. S2 \\ rotational angle of S4 Frame w.r.t. S3 \\ rotational speed of the turbine $(\mathrm{rad} / \mathrm{s})$ \\ azimuthal position (rad) \\ local blade element slope
}

\section{References}

Bathe, K.J., Finite Element Procedures in Engineering Analysis Prentice-Hall, Inc., 1982.

Benfield, W.A. and Hurda, R.F. Vibration Analysis of Structure by Component Mode Situation AIAA J., Vol. 9, No. 7, July 1971.

Garrad, A.D. and Quarton, D.C. Symbolic Computing as a Tool in Wind Turbine Dynamics Proc. European Wind Energy Conference, 22-26 October 1984, Hamburg, Germany, pp 295-301.

MSC/NASTRAN User's Manual, Vols. I and II, The MacNeal Schwendler Corp., 1981.

Kirchgäßner, B. ARLIS-A Program System For Aeroelastic Analysis of Rotating Linear System Proc. European Wind Energy Conference, 22-26 October 1984, Hamburg, Germany, pp 253-258.

Lobitz, D.W. and Ashwill, T.D. Aerolastic Effects in the Structural Dynamic Analysis of Vertical Axis Wind Turbines Sandia Report SAND 85-0957, April 1986.

Popelka, D. Aeroelastic Stability Analysis of a Darrieus Wind Turbine Sandia Report SAND 82-0672, February 1982.

Machielse, L.A.H. The $15 \mathrm{~m}$ Vertical Axis Wind Turbine Pionier I-Description and Experiments Proc. European Wind Energy Conf., 22-26 October 1984, Hamburg, Germany, pp 138-144.

Machielse, L.A.H., Rieffe, H.Ch. and P.M. Peeters Structural Vibration Frequencies and Drive Train Behavior of $15 \mathrm{~m}$ VAWT ECN-86-038. Ottens, H.H. Calculated Dynamic Behavior of the $15 \mathrm{~m}$ Vertical Axis Wind Turbine NLR Memorandum SC-81-044 U and Appendix (in Dutch).

Blackwell, B.F. and Reis, G.E. Blade Shape for a Troposkien Type of Vertical-Axis Wind Turbine Sandia Report SLA-74-0154, 1974.

El-Sayed, A.F.A.A. Aeroelastic Analysis of Vertical Axis Wind Turbines Proc. ISROMAC-3, Honolulu, Hawaii, 1990.

Users Guide to DAPST Vrije Universiteit Brussel Report, May 1985.

Meirovitch, L. Elements of Vibration Analysis McGraw-Hill Publishing Co., 1975.

Strickland, J.H. The Darrieus Wind Turbines: A Performance Prediction Model Using Multiple Streamtubes Sandia Report SAND 75-0431 October, 1975.

Sharpe; D.J. A Theoretical and Experimental Study of the Darrieus Vertical Axis Wind Turbine School of Mech., Aero. \& Prod. Engry Kingston Polytechnic Research Rep., Oct. 1977.

Templin, R.J. Aerodynamic Performance Theory for the NRC VerticalAxis Wind Turbine. Nat. Res. Council Canada, Laboratory Technical Report LTR-LA-160, June 1974. 
Simhan, K. A Review of Calculation Methods for the Determination of Performance Characteristics of Vertical Axis Wind Energy Converters with Special Reference to the Influence of Solidity and Starting Characteristics Proc. European Wind Energy Conf., Hamburg, Germany, October 1984.

Mandal, A.C. Aerodynamic and Design Analysis of Vertical Axis Darrieus Wind Turbines Ph.D. Thesis, Vrije Universiteit Brussel, Jan 1986.

Worstell, M.H. Aerodynamic Performance of the $17 \mathrm{~m}$ Diameter Darrieus Wind Turbine SAND 78-1737, 1978 and SAND 79-1753, 1979.
Muraca, R.J. and Guillotte, R.J. Wind Tunnel Investigation of 14 Vertical Axis Windmill NASA TM X-72663, 1976

South, P. and Rangi, R.S. A Wind Tunnel Investigation of 14 FT Diameter Vertical Axis Windmill Nat. Research Council Canada, Lab. Tech. Rep., LTR-LA-105, Sep., 1972.

Paraschivoiu, I. Aerodynamic Loads and Performance of the Darrieus Rotor J. Energy, Vol. 6, No. 6, pp 406-412, 1982.

Paraschivoiu, I. and Allet, A. Aerodynamic Analysis of the Darrieus Wind Turbine Including Dynamic-Stall Effects J. Propulsion and Power, Vol. 4, No. 5. Sept.-Oct., 1988, pp 472-477. 

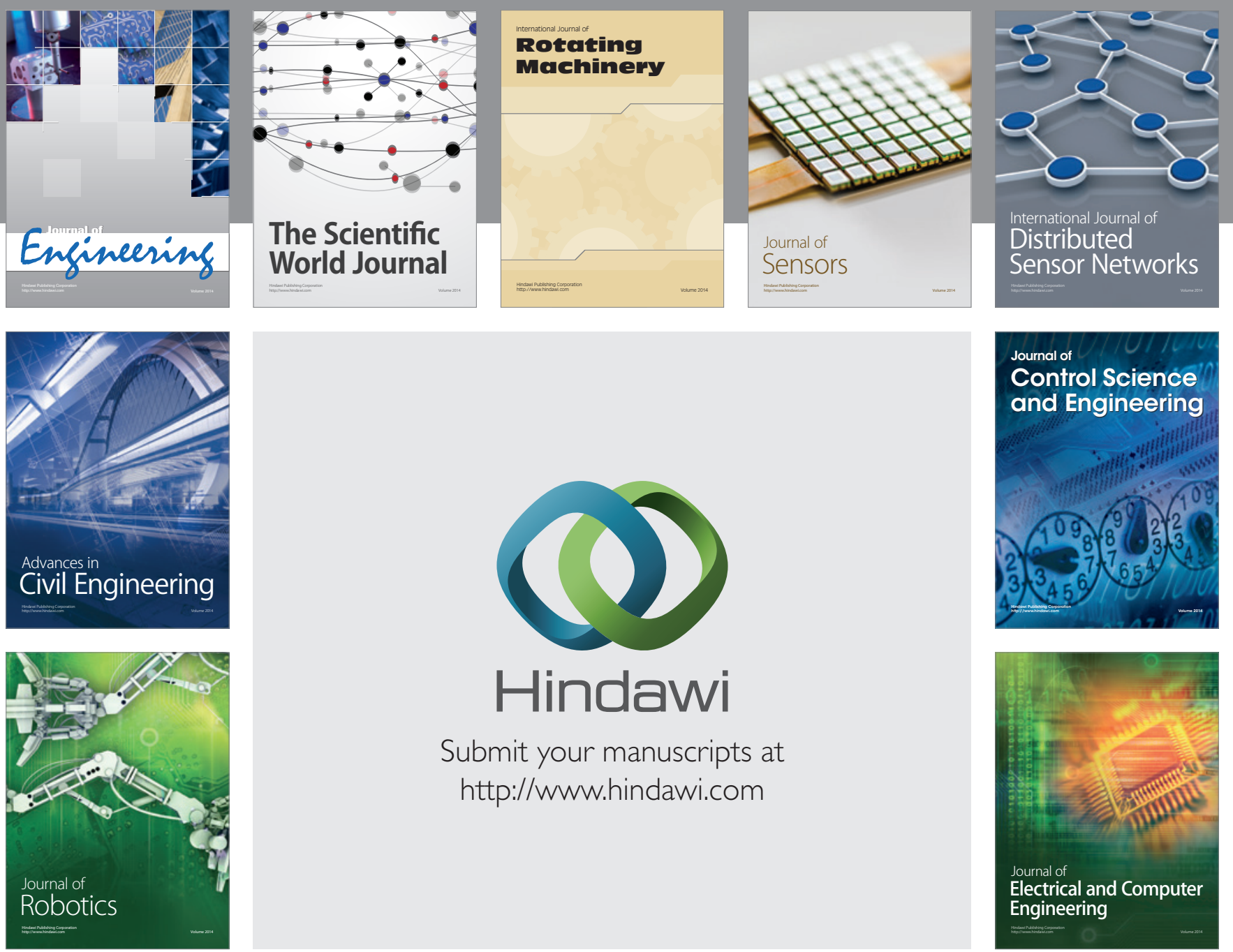

Submit your manuscripts at

http://www.hindawi.com
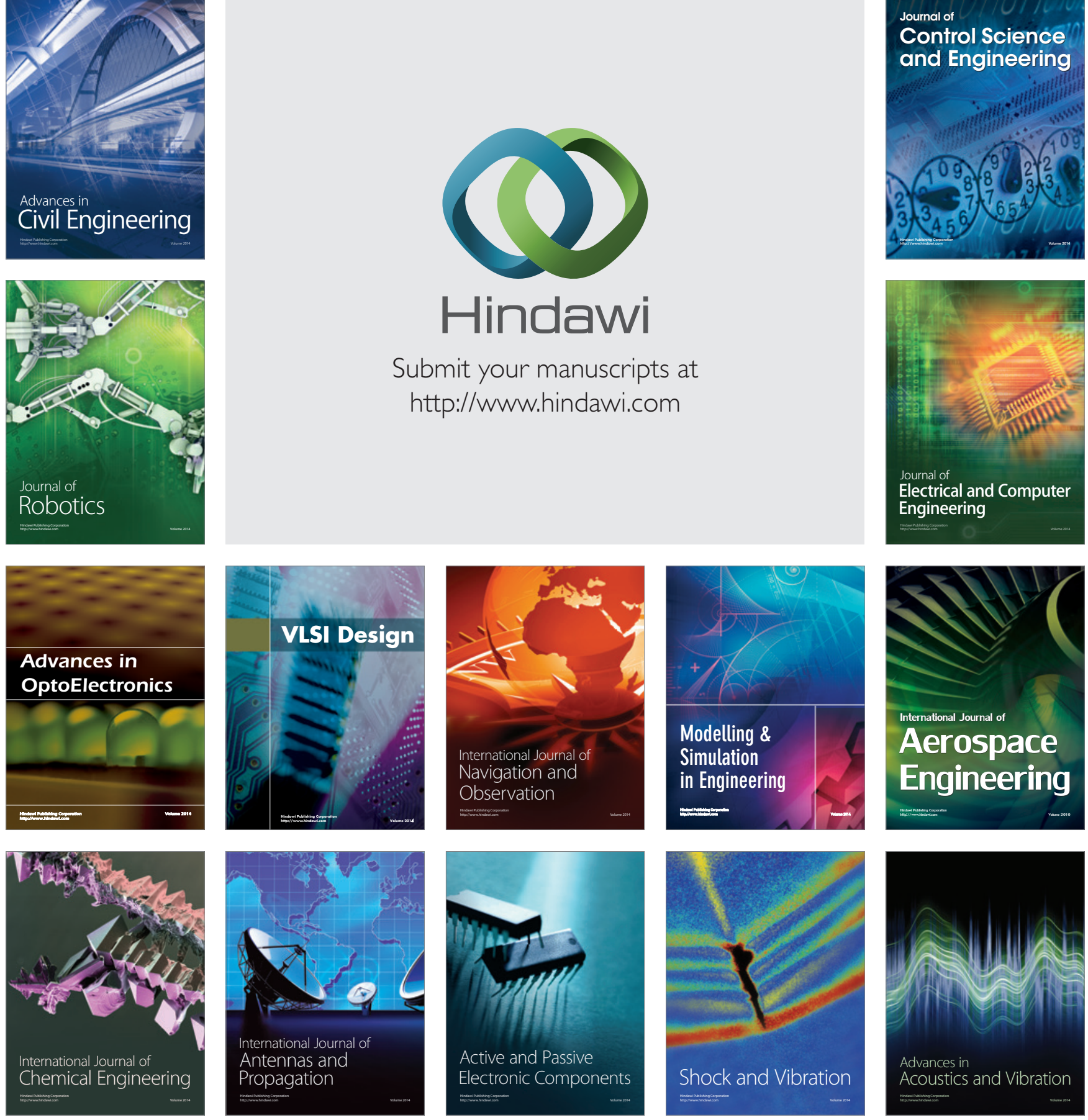